\title{
Intercell Interference Coordination through Limited Feedback
}

\author{
Lingjia Liu, ${ }^{1}$ Jianzhong (Charlie) Zhang, ${ }^{1}$ Jae-Chon Yu, ${ }^{2}$ and Juho Lee ${ }^{2}$ \\ ${ }^{1}$ Dallas Telecommunications R\&D Center, Samsung Telecommunications America, Richardson, TX 75082, USA \\ ${ }^{2}$ Standards and Industry Initiative (SII), Samsung Electronics, 416, Maetan-3dong, Yeongtong-gu, Suwon-si, \\ Gyeonggi-do 443-742, South Korea \\ Correspondence should be addressed to Lingjia Liu, lingjialiu@gmail.com
}

Received 3 August 2009; Accepted 2 November 2009

Academic Editor: Hongxiang Li

Copyright (๑) 2010 Lingjia Liu et al. This is an open access article distributed under the Creative Commons Attribution License, which permits unrestricted use, distribution, and reproduction in any medium, provided the original work is properly cited.

We consider the applications of multicell transmission schemes to the downlink of future wireless communication networks. A multicell multiple-input multiple output-(MIMOs) based scheme with limited coordination among neighboring base stations (BSs) is proposed to effectively combat the intercell interference by taking advantage of the degreesoffreedom in the spatial domain. In this scheme, mobile users are required to feedback channel-related information to both serving base station and interfering base station. Furthermore, a chordal distance-based compression scheme is introduced to reduce the feedback overhead. The performance of the proposed scheme is investigated through theoretical analysis as well as system level simulations. Both results suggest that the so-called "intercell interference coordination through limited feedback" scheme is a very good candidate for improving the cell-edge user throughput as well as the average cell throughput of the future wireless communication networks.

\section{Introduction}

Recent years have been marked by a soaring demand for network access. This trend is exemplified by the constant growth of wireless communication systems. The strong demand for network connectivity is partially fueled by new software applications and a widespread desire for real-time information access. Hence, future wireless communication networks will face the dual challenge of supporting large traffic volumes and providing reliable service to delaysensitive applications such as voice over IP (VoIP), videoconferencing, and online gaming. There are two performance measures that are crucial for wireless systems: average cell throughput and cell-edge user throughput [1]. Improving both of the performance measures becomes one of the major tasks of the next generation wireless communication systems. However, it is important to note that improving average cell throughput is a relatively easy task, while improving cell-edge user throughput becomes extremely demanding. This is because the average cell throughput can be improved using simple methods such as transmission power boosting. However, for cell-edge user throughput, these simple methods are not valid any more. Cell-edge users usually have relatively low received signal strength; furthermore, they do suffer from strong inter-cell interference. Transmission power boosting may increase the received signal strength, but it will also create stronger intercell interference to other cell's cell-edge users and hence reduce their throughput. Therefore, improving cell-edge user throughput becomes highly nontrivial. This is also part of the reasons why interference mitigation technologies for next generation wireless systems receive enormous attention in the standardization societies as well as in the research community [1-4].

In the wireless systems equipped with multiple transmit antennas, each cell applies a precoding vector on the transmit antennas to form a beam pointing towards targeted mobile stations (MSs). Current design of the wireless systems requires the scheduler at each cell to choose the precoding vector for beam-forming purely based on the wireless channel between the BS and the targeted MS [5]. Without taking into account which precoding vectors are used in 
the neighboring cells, the beams formed by different cells may randomly collide with each other, which results in substantial inter-cell interference for the cell-edge users.

In order to mitigate the interference to the cell-edge users and increase the system spectral efficiency, multi-cell MIMO is proposed as an enabling technology for future wireless systems $[6,7]$. In multi-cell MIMO, the network is required to process and transmit the data for an intended mobile user jointly from multiple geographically separated cells. This technology can greatly enhance the performance of cell-edge mobile users since it effectively changes the interference into useful signals. However, it requires the network to have access to the full channel station information (CSI) and requires the data for the intended mobile user to be available at all base stations. These two assumptions seem to be pretty restrictive in the practical wireless systems. Currently, only codebook-based feedback of CSI is widely adopted in the standards to reduce the uplink overhead $[5,8]$. Furthermore, practical issues such as backhaul delay and cost will limit the possibility of having one mobile user's data delivered to multiple base stations to perform joint processing. Therefore, it is also interesting to investigate interference mitigation schemes where the data for the intended mobile user is transmitted from a single serving cell. However, as opposed to the single cell operation, the scheduler should choose a precoding vector based on the link between the serving cell and the targeted MS together with the interference the serving cell may cause to the other cell's cell-edge users. Accordingly, we propose to jointly choose the precoding vectors among different cells to mitigate the inter-cell interference taking advantage of the spatial domain degrees of freedom introduced by MIMO systems. Only limited overhead control information is needed to enable this technology and each cell is able to choose his/her precoding vector in a distributed fashion. In other words, a central scheduler is not necessary for the proposed scheme. Both the analytical and simulation results suggest that the proposed scheme can significantly improve average cell throughput as well as the throughput of the cell-edge users.

The paper is structured as follows. Section 2 contains the system model. The theoretical foundation of the proposed inter-cell interference coordination schemes is illustrated in Section 3. Based on the derivation, we propose two different inter-cell interference coordination schemes in Section 4. We detail the simulation results in Section 5 and conclude in Section 6.

\section{System Model}

In this section, we start to analyze the throughput performance of cell-edge users. The typical scenario of two cell-edge users interfering with each other is illustrated in Figure 1. The corresponding system setup is that both of the base stations are communicating to the two corresponding serving mobile users simultaneously in the same frequency band. In Figure 1, BS1 is the serving cell for MS1 while BS2 is the serving cell for MS2. In this simple wireless system, assume that both MS1 and MS2 are cell-edge users and they are geometrically close to each other. The system described in Figure 1. is actually one of the worst interference cases for the cell-edge users because both users' performance are limited by the strong interference from the interfering cells. This fact can be seen most clearly from the expression of the received signal strength at each mobile user. To be specific, the received signals, $Y_{1}$ and $Y_{2}$, of MS1 and MS2 can be written as

$$
\begin{aligned}
& Y_{1}=H_{11} w_{1} X_{1}+H_{21} w_{2} X_{2}+N_{1}, \\
& Y_{2}=H_{12} w_{1} X_{1}+H_{22} w_{2} X_{2}+N_{2},
\end{aligned}
$$

where $H_{i j}$ denotes the channel gain from the $i$ th $\mathrm{BS}$ to the $j$ th MS, $w_{i}$ is the precoding vector used at BS $i, X_{i}$ is the vector of transmitted signal at BS $i$, and $N_{i}$ is the additive white Gaussian noise (AWGN) vector at MS $i$. The received signal of MS1, $Y_{1}$, suffers from the interference from BS2 $\left(H_{21} w_{2} X_{2}\right)$ and $Y_{2}$ suffers from the interference from BS1 $\left(H_{12} w_{1} X_{1}\right)$. The received Signal to Interference-plus-Noise Ratio (SINR) for MS1 and MS2 can then be expressed as

$$
\begin{aligned}
& \operatorname{SINR}_{1}=\frac{\left\|H_{11} w_{1}\right\|^{2} P_{1}}{\left\|H_{21} w_{2}\right\|^{2} P_{2}+N}, \\
& \operatorname{SINR}_{2}=\frac{\left\|H_{22} w_{2}\right\|^{2} P_{2}}{\left\|H_{12} w_{1}\right\|^{2} P_{1}+N},
\end{aligned}
$$

where $P_{i}$ is the transmitted power of $X_{i}$ at BS $i$, and $N$ is the noise power. In current LTE (Long Term Evolution) system [5], scheduler at BS1 chooses the precoding vector, $w_{1}$, purely based on the wireless channel between the BS1 and the targeted MS1, that is, $H_{11}$; while scheduler at BS2 chooses $w_{2}$ purely based on the channel from BS2 to MS2, that is, $H_{22}$. Since MS1 and MS2 are geographically close to each other, the channel gains from the BSs to the MSs are usually correlated. That is, $H_{11}$ and $H_{12}$ are correlated, and $H_{21}$ and $H_{22}$ are correlated. Therefore, the precoding vector, $w_{1}$, which maximizes $H_{11} w_{1} X_{1}$ may also produce large magnitude of $H_{12} w_{1} X_{1}$ which is the interference from BS1 to MS2. Increasing the transmission power will also increase the interference to other cell's cell-edge users in a linear way. Since MS1 and MS2 are both cell-edge users, the received signal strength will be comparable to the received interference strength. Therefore, $\mathrm{SINR}_{1}$ and $\mathrm{SINR}_{2}$ will be normally below $0 \mathrm{~dB}$. The fact that both the mobile users experience very low SINR limits the performance of the whole system and cannot be resolved by simply increasing the transmit power of BS1 and BS2.

\section{Theoretical Foundation}

In the previous section, we have developed some critical understandings of the interference for the cell-edge users. In this section, we will analyze fundamentals of "Inter-cell Interference Coordination through limited feedback" and show how it will improve the throughput of cell-edge users.

Even though the inter-cell interference cannot be effectively eliminated by increasing or reducing the total transmission power, it is interesting to note that it can actually be greatly reduced through optimizing over the precoding 


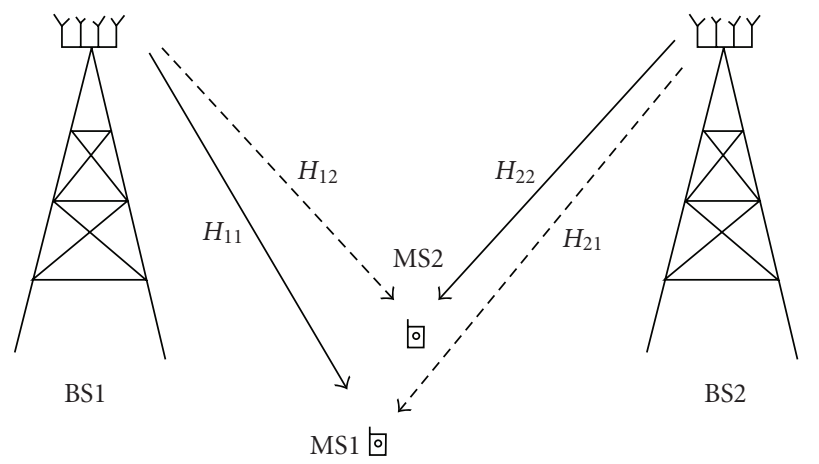

FIGURE 1: System model of two interfering cell-edge users.

vectors in the spatial domain. For the wireless system shown in Figure 1, it can be seen from (2) that the $\mathrm{SINR}_{1}$ and SINR $_{2}$ are functions of $w_{1}$ and $w_{2}$. In other words, we can optimize over $w_{1}$ and $w_{2}$ to improve both $\mathrm{SINR}_{1}$ and $\mathrm{SINR}_{2}$. Furthermore, for a wireless system equipped with multiple transmit antennas the inter-cell interference can be partially or completely cancelled by applying different precoding vectors at different base stations. This can be achieved by exploring the additional degrees of freedom offered by multiple transmit antennas in the spatial domain.

In [9], an optimal noncooperative zero-forcing beamforming is proposed. A mobile user is required to feedback the precoding vector to the serving cell taking into account the effects of the interference channel. In this way, the transmitted signal from the serving cell can effectively "avoid" the interference from other cells. Assume that MS1 has the ability to estimate the interference channel $\left(H_{21} w_{2}\right)$ from base Station 2; mathematically, MS1 will compute the precoding vector based on

$$
w_{1}^{\prime}=\underset{w_{1} \in \Gamma}{\arg \max } \frac{\left\|H_{11} w_{1}\right\|^{2} P_{1}}{\left\|H_{21} w_{2}\right\|^{2} P_{2}+N},
$$

where $\Gamma$ stands for the codebook. This scheme performs well under the assumption that there will be no communication between the cells. However, for the wireless system where the channel-related information can be exchanged over the network, it is strictly suboptimal. Accordingly, the optimal way is to jointly choose the precoding vectors in (2).

Before going to the details of the proposed communication scheme let us take a deeper look at the interference of MS1 from BS2 in the system depicted in Figure 1. Assume that the number of transmit antennas at the BS is $N_{T}$ and the number of receive antennas at the MS is $N_{R}$; the channel gain matrix $H_{21}$ then becomes an $N_{R}$ by $N_{T}$ matrix. In the wireless systems the number of transmit antennas at the base stations is always greater than or equal to the number of receive antennas at the mobile users; therefore, we can safely assume
$N_{R} \leq N_{T}$ throughout the paper. Applying the singular value decomposition (SVD) [10] to $H_{21}$, we have

$$
H_{21}=U \Lambda V=U \times\left[\begin{array}{cccc}
\lambda_{1} & & & 0 \\
& \ddots & & \vdots \\
& & \lambda_{N_{R}} & 0
\end{array}\right]_{N_{R} \times N_{T}} \times V,
$$

where $U$ is an $N_{R}$ by $N_{R}$ unitary matrix, $V$ is an $N_{T}$ by $N_{T}$ unitary matrix, and $\lambda_{1}$ through $\lambda_{N_{T}}$ are the singular values of the channel gain matrix $H_{21}$. After applying the precoding vector, the interference seen at MS1 can be expressed as

$$
\begin{aligned}
H_{21} w_{2} X_{2} & =U \Lambda V w_{2} X_{2} \\
& =U \times\left[\begin{array}{cccc}
\lambda_{1} & & & 0 \\
& & & \vdots \\
& & & \\
& & \lambda_{N_{R}} & 0
\end{array}\right]_{N_{R} \times N_{T}} \times V \times w_{2} \times X_{2} .
\end{aligned}
$$

Let

$$
X_{2}^{\prime}=V \times w_{2} \times X_{2}
$$

which is an $N_{T}$ by 1 vector. Accordingly, the interference from BS2 which is seen at MS1 can be rewritten in the form of

$$
H_{21} w_{2} X_{2}=U \times\left[\begin{array}{cccc}
\lambda_{1} & & & 0 \\
& \ddots & & \vdots \\
& & \lambda_{N_{R}} & 0
\end{array}\right]_{N_{R} \times N_{T}} \times X_{2}^{\prime} .
$$

As long as we choose the precoding vector, $w_{2}$, such that $X_{2}^{\prime}$ satisfies the condition,

$$
X_{2}^{\prime}=V \times w_{2} \times X_{2}=\left[\begin{array}{llllll}
0 & \cdots & 0 & x_{1} & \cdots & x_{N_{T}-N_{R}}
\end{array}\right]^{T},
$$

the interference seen at MS1, $H_{21} w_{2} X_{2}$, will be strictly zero. That is,

$$
\begin{aligned}
& H_{21} w_{2} X_{2}=U \times\left[\begin{array}{cccc}
\lambda_{1} & & & 0 \\
& \ddots & & \vdots \\
& & \lambda_{N_{R}} & 0
\end{array}\right]_{N_{R} \times N_{T}} \times\left[\begin{array}{c}
0 \\
\vdots \\
0 \\
x_{1} \\
\vdots \\
x_{N_{T}-N_{R}}
\end{array}\right] \\
& =\left[\begin{array}{c}
0 \\
\vdots \\
0
\end{array}\right]_{N_{R} \times 1} .
\end{aligned}
$$

Note that $x_{1}$ and $x_{2}$ can be arbitrary values and the precoding vectors satisfying (8) are not unique. This result indicates 
that there exists a set of precoding vectors at BS2 which will cause no interference to MS1 if we are allowed to choose precoding vectors freely. Furthermore, this set of precoding vectors lies in the null space of the interfering channel matrix. Similarly, the same result will apply to the received signal of MS2; that is, a set of precoding vectors at BS1 will cause no interference to MS2. This result is true as long as the number of transmit antennas at BS is larger than that at the MS which means that the null space of the interfering channel matrix is not empty. For the case where we have to select the precoding vectors from a predetermined set like in the LTE systems [5], there might be no precoding vectors satisfying the condition in (8). In this situation there always exists a precoding vector which creates least interference among all the available precoding vectors. By using this precoding vector, we can make sure that the interference created to the other cell is minimal within the predetermined precoding vector set.

Forcing the inter-cell interference to be zero or minimal is a very restrictive condition and greatly reduces the choice of the precoding vectors. For example, for an $N_{T}$ by $N_{R}$ wireless system, the precoding vectors satisfying (8) only spans $N_{T}$ $-N_{R}$ dimensions of the overall spatial domain which has a total dimension of $N_{T}$. Therefore, we introduce a parameter, SINR_thd, to relax the requirements of the interference seen by each MS. To be specific, $w_{2}$ is chosen to satisfy the following condition:

$$
w_{2}=\underset{w \in \Omega}{\arg } \frac{\left\|H_{11} w_{1}\right\|^{2} P_{1}}{\left\|H_{21} w\right\|^{2} P_{2}+N} \geq \text { SINR_thd, }
$$

where $\Omega$ is the set of all precoding vectors. Equation (10) means that $w_{2}$, when used at BS2, will introduce tolerable interference to MS1. Note that SINR_thd plays a crucial role in the precoding vector selection. When this threshold is large, more restrictive constraints are put on BS2's interference to MS1, which means that less number of precoding vectors will be used for BS2. In this scenario, interference can be greatly reduced but the multi-user diversity also reduced due to the restrictive selection of the precoding vectors at BS2. When this threshold is small, BS2 will have more freedom to choose the precoding vectors thus increasing multi-user diversity. However, the interference from BS2 to MS1 can still be large due to the loose condition of the SINR threshold. In a way, this threshold triggers a tradeoff between multi-user diversity and interference mitigation. Interestingly, the condition expressed in (8) is actually the special case when

$$
\text { SINR_thd }=\frac{\left\|H_{11} w_{1}\right\|^{2} P_{1}}{N} .
$$

\section{Intercell Interference Coordination through Limited Feedback}

Motivated by the elegant results shown in Section 3, we start to investigate on practical interference mitigation schemes through limited coordination. From the analysis we know that each BS has a set of precoding vectors that will cause controlled interference to the cell-edge users in the adjacent cells through parameter SINR_thd. Throughout this paper, we call this set of precoding vectors as "the recommended set." Therefore, if a BS can choose a precoding vector within this recommended set to maximize his/her SINR to the targeted MS, the inter-cell interference will be greatly mitigated. Accordingly, the cell-edge user throughput will be significantly improved. However, one question remains: how does the BS know about the set of recommended precoding vectors. It is interesting to note that the condition shown in (10) can actually be tested at each MS. Therefore, each MS can feedback the recommended set of precoding vectors to the interfering cell which will cause tolerable interference to the interfering cells.

Feeding back the whole set of recommended precoding vectors will cause too much signaling overhead for the system. Therefore, we must further optimize the feedback information to reduce the system overhead. Note that the recommended set of precoding vectors contains all the precoding vectors satisfying (10). That is, $w_{2}$ belongs to the recommended set if and only if

$$
\left\|H_{21} w_{2}\right\|^{2} \leq\left(\frac{\left\|H_{11} w_{1}\right\|^{2} P_{1}}{\text { SINR_thd }}-N\right) / P_{2}=\alpha .
$$

In order to reduce the feedback overhead of the coordination scheme, we can take a deeper look at the necessary and sufficient condition of the recommended precoding vector in (12). The left-hand side (LHS) of above inequality is actually related to a distance measure between $H_{21}$ and $w_{2}$. Therefore, (12) suggests that a distance measure threshold together with a reference precoding vector can be used to completely characterize the set of recommended precoding vectors. This result can be seen most clearly through a simple example. Assume that we have an $N_{T}$ by 1 wireless system, that is, $N_{T}$ transmit antennas at the BS and 1 receive antenna at the MS. For this simple system, the channel matrix $H_{21}$ becomes a 1 by $N_{T}$ vector which can be written as the hermitian of a $N_{T}$ by 1 vector. That is, $H_{21}=w^{*}$, where $w$ is a $N_{T}$ by 1 vector. Therefore, the LHS of (12) can be rewritten as

$$
\left\|H_{21} w_{2}\right\|^{2}=\left\|w^{*} w_{2}\right\|^{2} \leq \alpha .
$$

The above expression is actually the cross-correlation between two $N_{T}$ by 1 vectors. Since both $w$ and $w_{2}$ are unitary, we can further rewrite (13) into

$$
d_{\text {chordal }}\left(w, w_{2}\right)=\sqrt{1-\left\|w^{*} w_{2}\right\|^{2}} \geq \sqrt{1-\alpha},
$$

where $d_{\text {chordal }}\left(w, w_{2}\right)$ stands for the chordal distance between $w$ and $w_{2}$ [11]. In this example, the distance measure is the chordal distance and the reference precoding vector is the precoding vector $w=H_{12}^{*}$.

In the case where one particular cell receives multiple recommended sets from various cells, the scheduler should be able to choose one of the requests based on overall system throughput. To facilitate the choice at the scheduler, each MS should also report the SINR or channel quality improvement when the recommended set of precoding vectors is used 


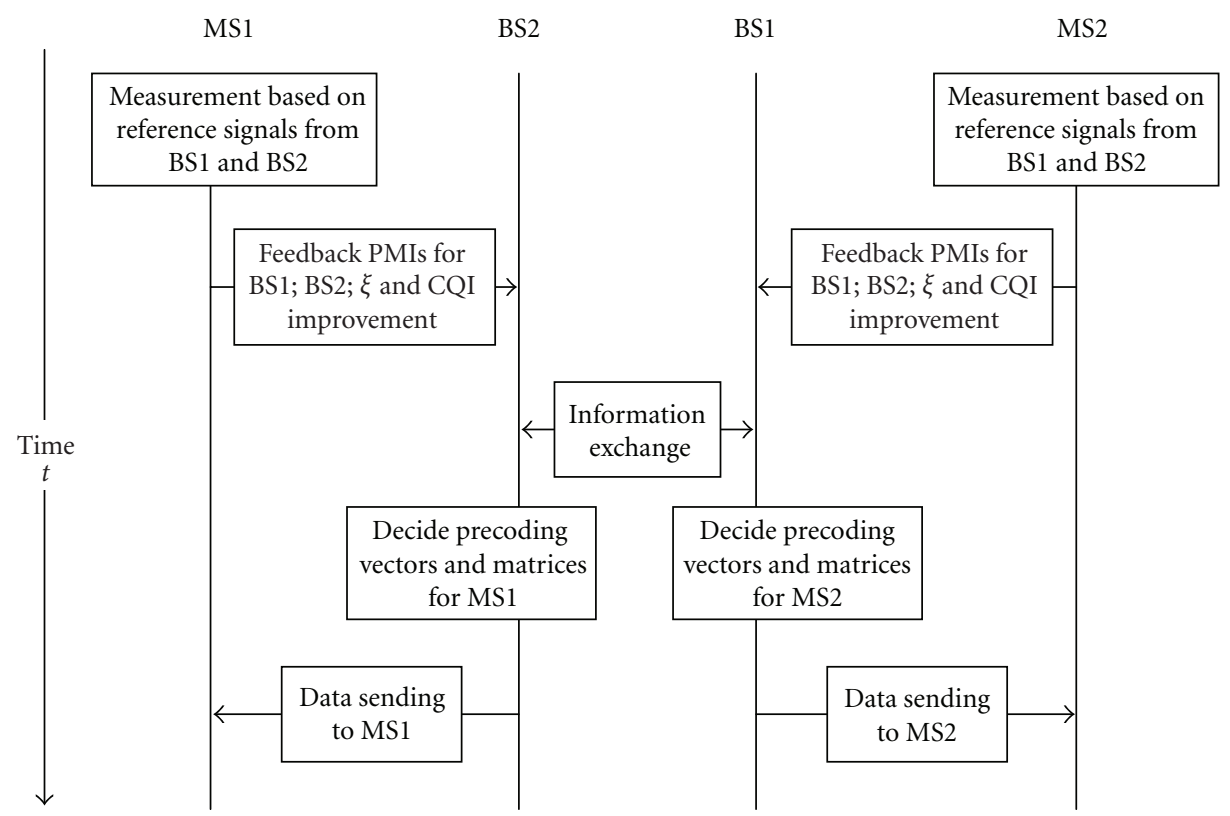

FIGURE 2: Time line of proposed inter-cell interference coordination through limited feedback.

at the interfering cells. Accordingly, when facing multiple requests at one cell, the scheduler should accept the request with highest the SINR or channel quality improvement so that the system performance improves most.

Based on all the results and understandings, the overall procedure of the proposed "Inter-cell interference coordination through limited feedback" scheme is listed as follows.

Step 1. Each MS measures the channel from the serving cell as well as the interfering cells.

Step 2. Each MS obtains the feedback information for interfering links. The feedback information contains what follows:

(i) reference precoding vectors (PMI) from the interfering cells,

(ii) a distance measure threshold indicating the sets of precoding vectors,

(iii) precoding vector and channel quality index (CQI) for the serving cell.

Step 3. Each MS obtains the measure for performance improvement:

(i) SINR improvement when the recommended set of precoding vectors is used at the interfering cells.

Step 4. Each MS feeds back the information to serving cell as well as interfering cells.

Note that in this mode of operation, the MS can send all the feedback information to the serving cell relying on the serving cell to relay all the related information to the

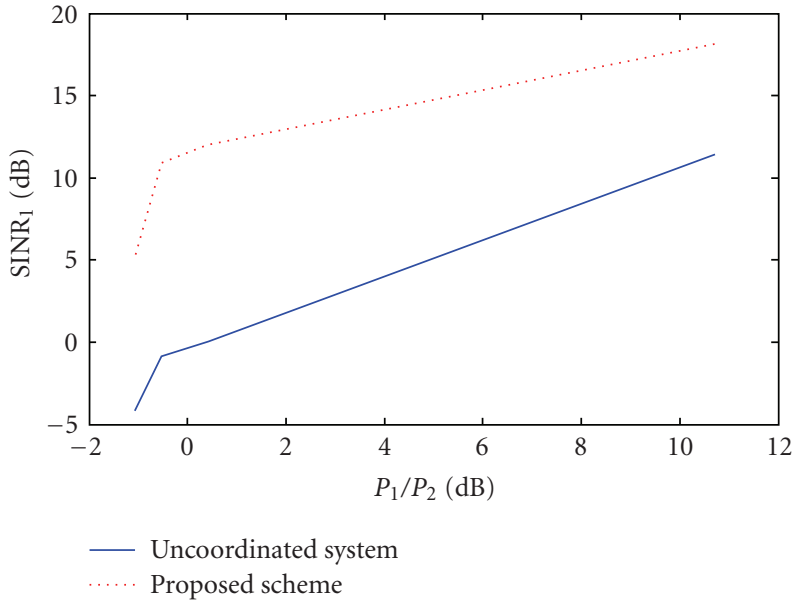

Figure 3: SINR improvement.

interfering cells. Also, the MS can choose to feedback the information to the intended destination directly. That is, the reference precoding vector together with the threshold can be sent back to the interfering cells from the MS directly.

Step 5. Serving cell the interfering cells choose corresponding precoding vectors to serve their targeted users.

In this mode of operation, interfering cells are suggested to choose the precoding vector which maximizes his/her own serving MS's throughput within the recommended set if no central scheduler is present. In the case where a central scheduler is present, the precoding vectors for the serving MS are decided jointly across all the serving cells by the central scheduler. 


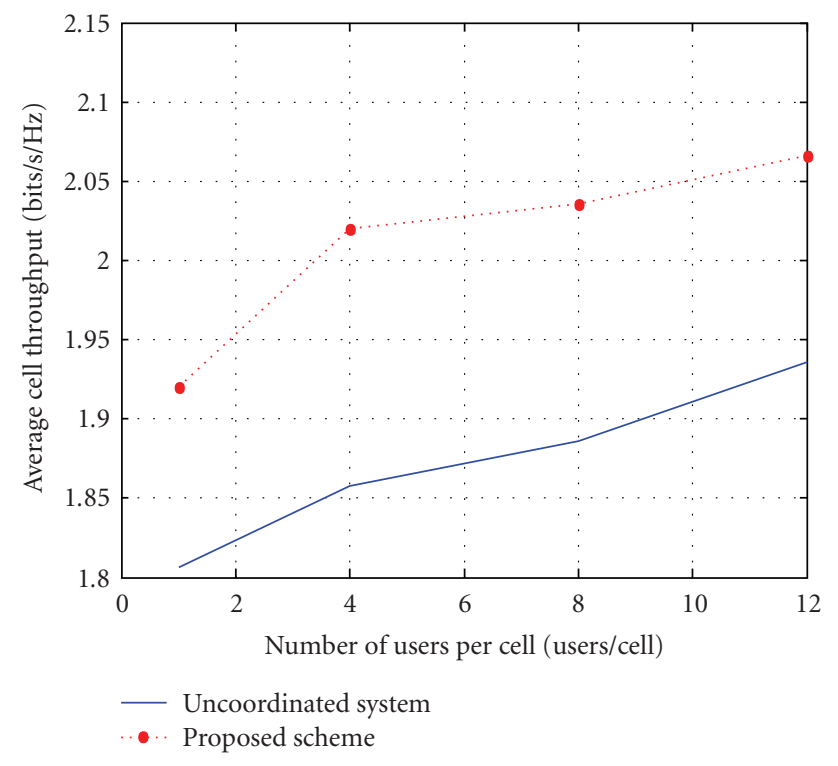

FIgURE 4: Average cell throughput comparison.

The timeline of the proposed interference coordination scheme can be shown in Figure 2. In the proposed block diagram, $\xi$ stands for the distance measure threshold summarizing the recommended precoding vector sets.

\section{Simulation Results}

The performance of the proposed coordination scheme can be evaluated through link level simulation on the SINR improvement as well as the system level simulation on average cell throughput together with cell-edge user throughput (5\% user throughput). The system parameters for the simulations strictly follow the evaluation methodology proposed by the 3GPP community [12]. Furthermore, according to the current LTE specification, we assume that there are 4 transmit antennas at the base station and 2 receive antennas at the mobile user.

The link level simulation result is contained in Figure 3. Figure 3 compares the SINR of the "Inter-cell Interference coordination with limited feedback" and that of the uncoordinated system. $P_{1}$ is the average transmission power of base station 1 and $P_{2}$ is the average transmission power of base station 2. In this simulation, we assume that the interfering cell always accepts the recommendation from MS1 and the channel feedback is based on LTE codebook. It can be seen that there is a large improvement in terms of SINR gains of the cell-edge users. This performance gain is achieved by adding a little overhead (a message contains the set information) compared to current LTE system.

The SINR improvement shown in the link level simulation is somewhat biased in the sense that the hit of MS2's throughput is not shown. Since the coordination will limit the choice of precoding vectors at BS2, the throughput of MS2 will be affected. In order to take a more complete picture of the system, we conduct system level simulation. The system level simulation results for average cell throughput

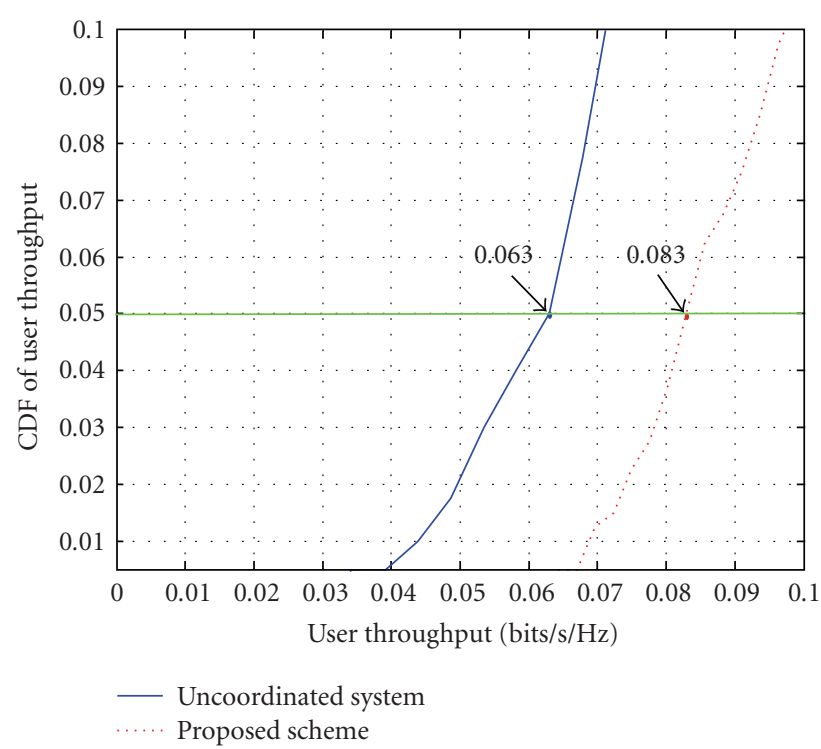

FIGURE 5: Cell-edge user throughput comparison.

and cell-edge user throughput based on SINR_thd $=0 \mathrm{~dB}$ are shown in Figures 4 and 5, respectively.

In the system level simulation, we assume that all the base stations accept the recommended set and choose the precoding vectors within the set. Figures 4 and 5 suggest that the improvement in average cell throughput can be as large as $10 \%$ while the improvement in 5\% sector throughput (cell-edge user throughput) can be as large as 30\%. This is because by adopting the recommended set, both received signal strength and received interference strength reduced. The overall SINR again is not significant for the cell-center users while it is huge for the cell-edge users (the limiting factor in SINR for cell-edge UE is interference). Both results suggest that the proposed interference mitigation scheme is extremely efficient for combating the inter-cell interference especially for cell-edge users.

\section{Conclusion}

Multi-cell MIMO is believed to be one of the enabling technologies in next generation wireless systems. To be specific, the downlink multi-cell MIMO transmission is mainly characterized into two classes [13] in the LTE-A standards: coordinated scheduling and joint transmission.

In the class of joint transmission, data to single MS is simultaneously transmitted from multiple BSs to improve the received signal quality. It has been shown in [6] that this operation mode can significantly increase average cell throughput as well as cell-edge user throughput. However, this scheme requires data to be shared among various cells and requires the network to have the full CSI. In this paper, we investigate schemes falling in the class of coordinated scheduling where data to single MS is instantaneously transmitted from one BS. It is shown that a huge SINR improvement as well as a large throughput increase can be achieved through the proposed scheme. The gains are 
achieved by using simple codebook-based channel feedback schemes and are crucial for cell-edge users. This gain is realized through taking advantage of the additional degrees of freedom from the spatial domain. Furthermore, a distance measure threshold-based technology is applied to further reduce the signaling overhead of the proposed scheme. Since the proposed scheme does not need to share data among different BSs and hence reduces the cost of coordination, we do believes this is a promising technology for interference mitigation in future wireless systems such as LTE-Advanced.

\section{Acknowledgment}

The authors would like to thank Dr. Farooq Khan, Mr. Zhouyue (Jerry) Pi, and Dr. Donghee Kim for useful discussions.

\section{References}

[1] 3GPP, "Feasibility study for further advancements for EUTRA (LTE-Advanced)," Tech. Rep. TR36.912, 3GPP, Valbonne, France.

[2] A. Jovicic, H. Wang, and P. Viswanath, "On network interference management," submitted to IEEE Transactions on Information Theory.

[3] H. Li, B. Liu, and H. Liu, "Transmission schemes for multicarrier broadcast and unicast hybrid systems," IEEE Transactions on Wireless Communications, vol. 7, no. 11, pp. 4321-4330, 2008.

[4] H. Li, S. U. Khan, and H. Liu, "Broadcast network coverage with multi-cell cooperation," to appear in The International Journal of Digital Multimedia Broadcasting.

[5] 3GPP, "Evolved Universal Terrestrial Radio Access (E-UTRA); physical channels and modulation," Tech. Rep. TS36.211. v 8.6.0, 3GPP, Valbonne, France.

[6] S. Jing, D. N. C. Tse, J. B. Soriaga, J. Hou, J. E. Smee, and R. Padovani, "Multicell downlink capacity with coordinated processing," EURASIP Journal on Wireless Communications and Networking, vol. 2008, Article ID 586878, 19 pages, 2008.

[7] S. A. Jafar and S. Shamai, "Degrees of freedom region of the MIMO X channel," IEEE Transactions on Information Theory, vol. 54, no. 1, pp. 151-170, 2008.

[8] "IEEE 802.16 WirelessMAN Standard: Myths and Facts," ieee802.org.

[9] J. Kotecha and J. Mundarath, "Non-collaborative zerofForcing beamforming in the presence of co-channel interference and spatially correlated channels," in Proceedings of the 66th IEEE Vehicular Technology Conference (VTC '07), pp. 591-595, Baltimore, Md, USA, 2007.

[10] R. Horn and C. Johnson, Matrix Analysis, Cambridge University Press, Cambridge, UK, 1994.

[11] D. J. Love and R. W. Heath Jr., "Limited feedback unitary precoding for spatial multiplexing systems," IEEE Transactions on Information Theory, vol. 51, no. 8, pp. 2967-2976, 2005.

[12] 3GPP, "Physical layer aspect for evolved Universal Terrestrial Radio Access (UTRA)," Tech. Rep. TR25.814. v7.1.0, 3GPP, Valbonne, France.

[13] 3GPP, "Evolved Universal Terrestrial Radio Access (E-UTRA); further advancements for E-UTRA Physical layer aspects," Tech. Rep. TR36.814. v1.0.0, 3GPP, Valbonne, France. 

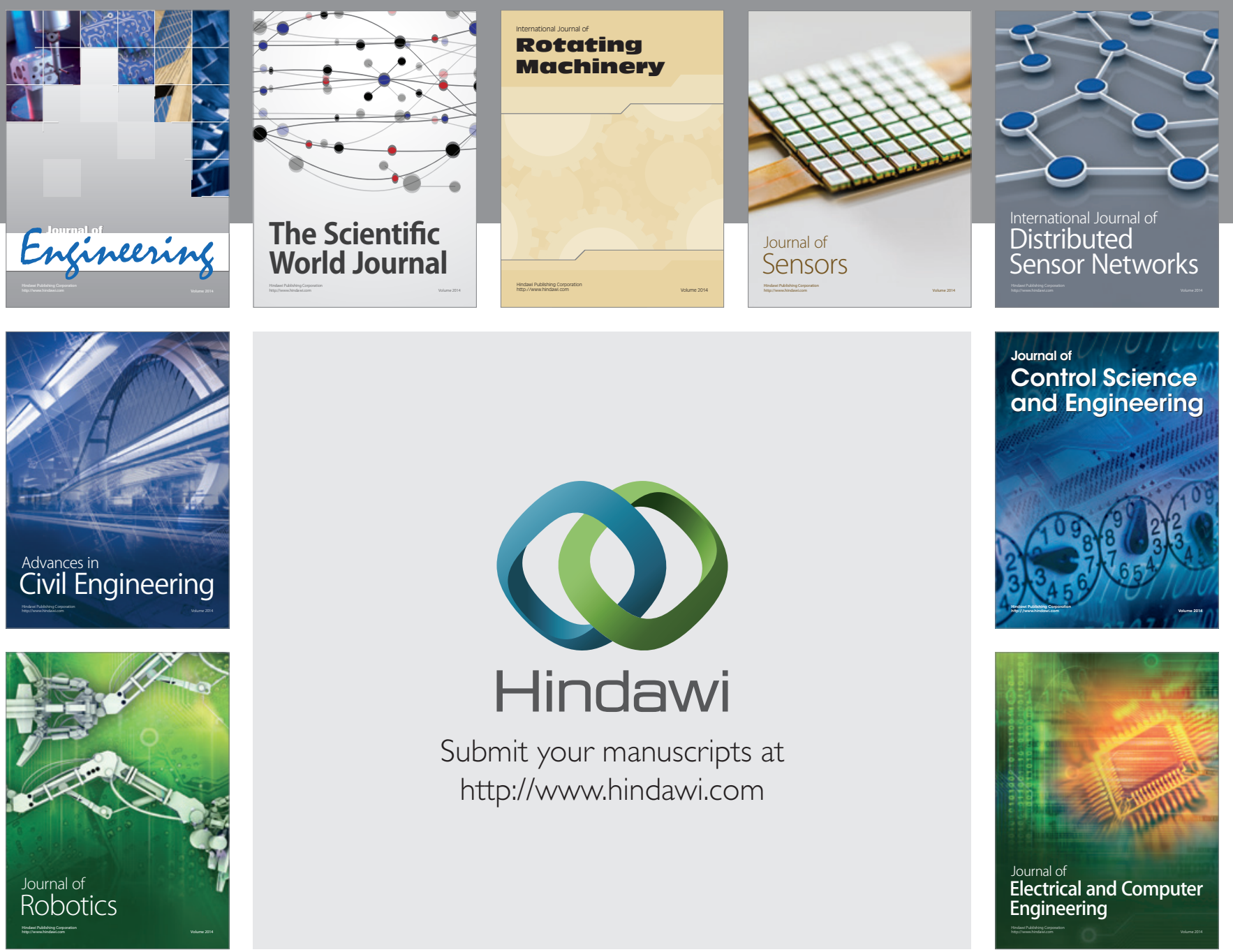

Submit your manuscripts at

http://www.hindawi.com
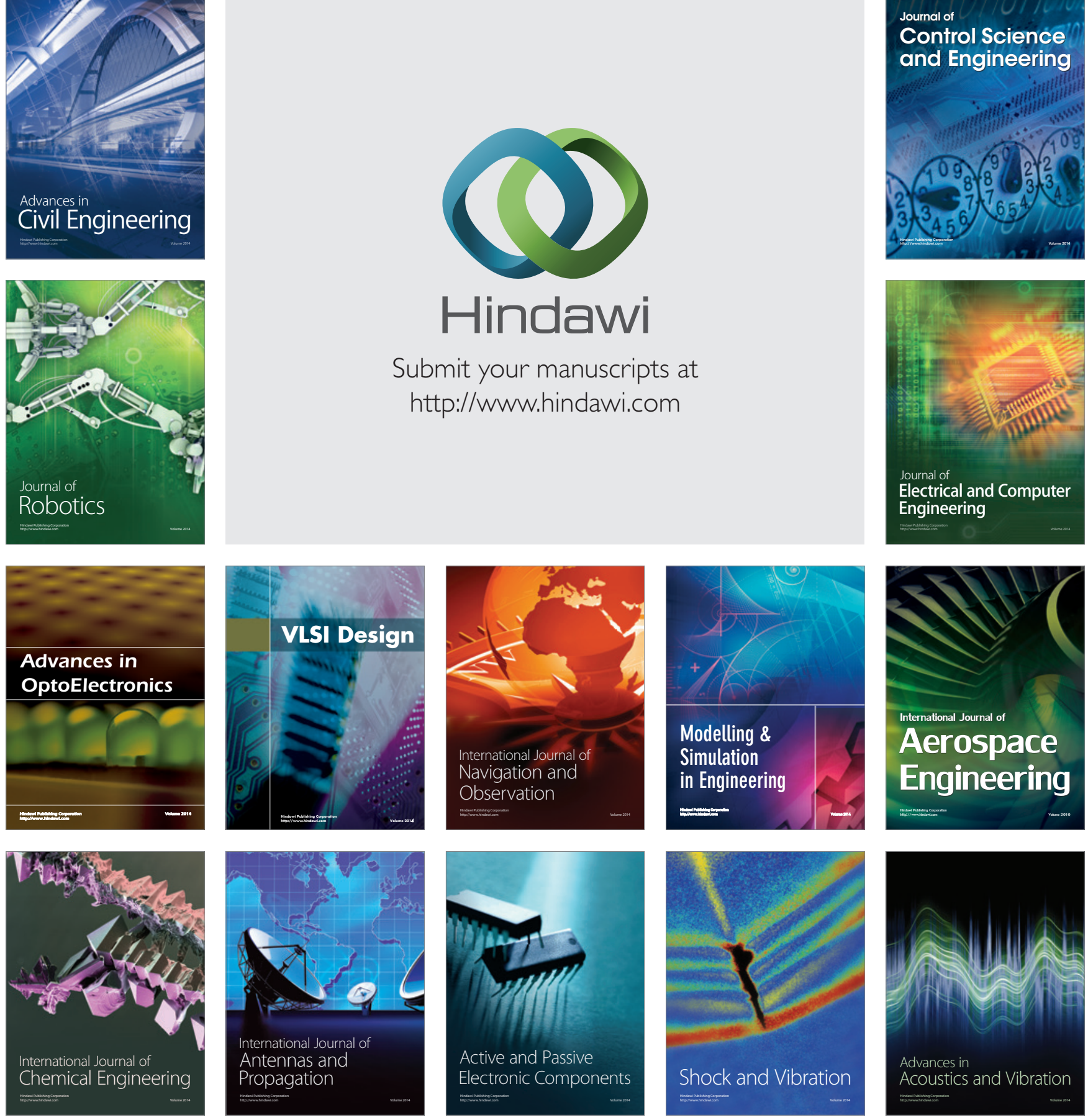